\title{
Unraveling the rapid radiation of crested newts (Triturus cristatus superspecies) using complete mitogenomic sequences
}

Ben Wielstra ${ }^{1,2^{*}}$ and Jan W Arntzen ${ }^{2}$

\begin{abstract}
Background: The rapid radiation of crested newts (Triturus cristatus superspecies) comprises four morphotypes: 1) the T. karelinii group, 2) T. carnifex - T. macedonicus, 3) T. cristatus and 4) T. dobrogicus. These vary in body build and the number of rib-bearing pre-sacral vertebrae (NRBV). The phylogenetic relationships of the morphotypes have not yet been settled, despite several previous attempts, employing a variety of molecular markers. We here resolve the crested newt phylogeny by using complete mitochondrial genome sequences.

Results: Bayesian inference based on the mitogenomic data yields a fully bifurcating, significantly supported tree, though Maximum Likelihood inference yields low support values. The internal branches connecting the morphotypes are short relative to the terminal branches. Seen from the root of Triturus (NRBV = 13), a basal dichotomy separates the T. karelinii group (NRBV $=13$ ) from the remaining crested newts. The next split divides the latter assortment into T. carnifex - T. macedonicus (NRBV = 14) versus T. cristatus (NRBV $=15$ ) and T. dobrogicus (NRBV $=16$ or 17 ).

Conclusions: We argue that the Bayesian full mitochondrial DNA phylogeny is superior to previous attempts aiming to recover the crested newt species tree. Furthermore, our new phylogeny involves a maximally parsimonious interpretation of NRBV evolution. Calibrating the phylogeny allows us to evaluate potential drivers for crested newt cladogenesis. The split between the T. karelinii group and the three other morphotypes, at ca. 10.4 $\mathrm{Ma}$, is associated with the separation of the Balkan and Anatolian landmasses (12-9 Ma). No currently known vicariant events can be ascribed to the other two splits, first at ca. $9.3 \mathrm{Ma}$, separating T. carnifex - T. macedonicus, and second at ca. 8.8 Ma, splitting T. cristatus and T. dobrogicus. The crested newt morphotypes differ in the duration of their annual aquatic period. We speculate on the role that this ecological differentiation could have played during speciation.
\end{abstract}

\section{Background}

Understanding the temporal framework in which species have originated is fundamental to historical biogeography and evolutionary studies. However, obtaining a reliable phylogeny for a model system is not always straightforward. Rapid radiations are notoriously difficult in this respect, and the older the radiation, the more pronounced the problem will be [1]. The crested newt Triturus cristatus superspecies (Amphibia: Salamandridae), distributed in Europe and adjacent Asia (Figure 1),

\footnotetext{
* Correspondence: ben.wielstra@ncbnaturalis.nl

'Netherlands Centre for Biodiversity - NCB Naturalis, P. O. Box 9517, 2300 RA Leiden, The Netherlands

Full list of author information is available at the end of the article
}

is an example of a relatively old, rapid radiation, for which it has proved problematic to obtain a resolved phylogenetic tree.

The crested newt superspecies encompasses four morphological groups, hereafter referred to as 'morphotypes'. Ordered from a stocky build with sturdy limbs, to slender with small limbs, via two intermediate stages, these are: 1) the T. karelinii group, 2) T. carnifex - T. macedonicus, 3) T. cristatus and 4) T. dobrogicus. The morphotypes are characterized by discrete differences in the number of rib-bearing pre-sacral vertebrae (NRBV) [2]. The typical NRBV count is 13 for the T. karelinii group, 14 for $T$. carnifex - T. macedonicus, 15 for T. cristatus and 16 or 17 for $T$. dobrogicus. The marbled newts, T. marmoratus

\section{Biomed Central}




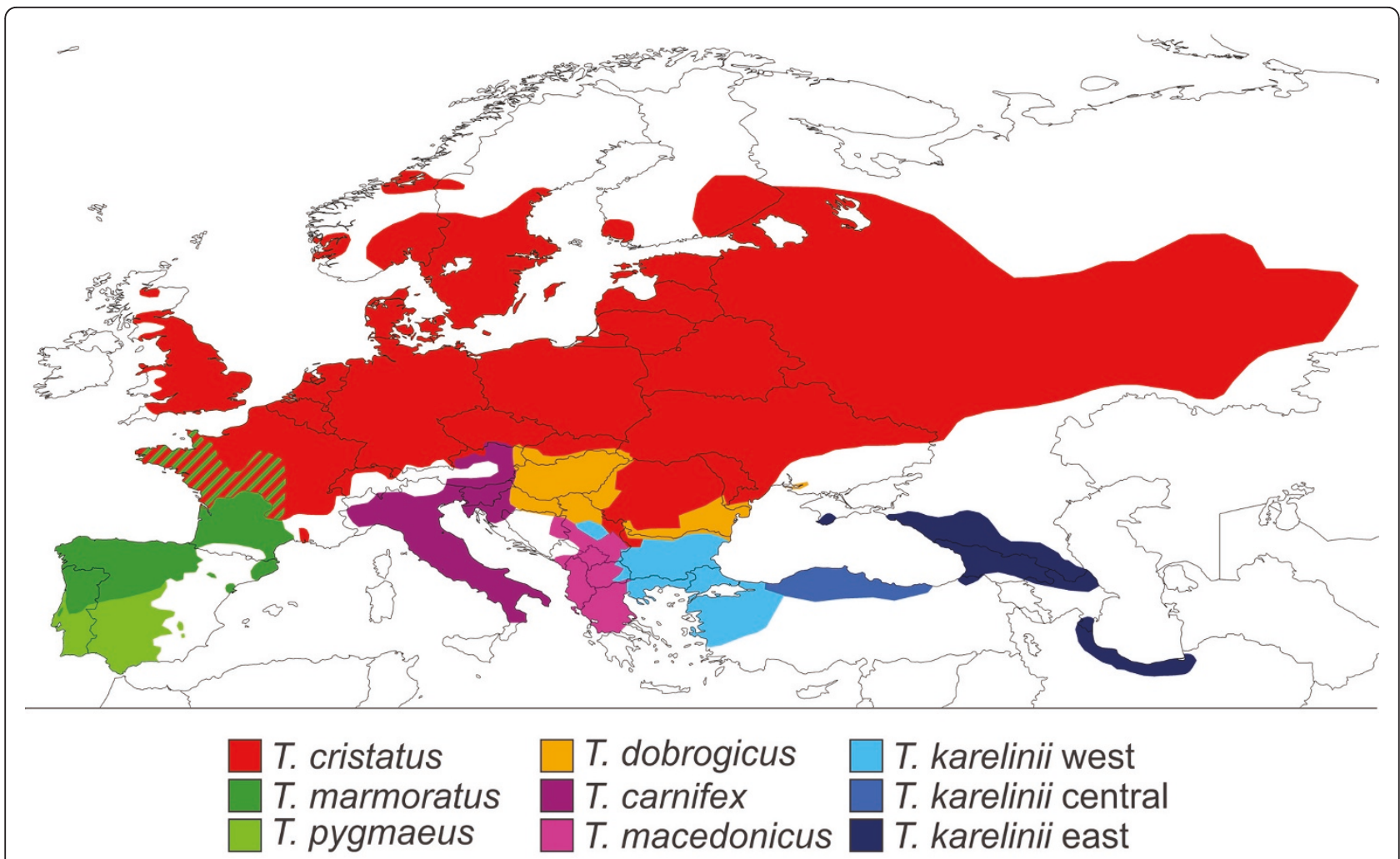

Figure 1 The distribution of the genus Triturus. Shown are the ranges for all the different species; the range of the T. karelinii group is partitioned according to the three distinct mitochondrial DNA clades the group is composed of (cf. [16]). Note the partially overlapping ranges of the crested newt T. cristatus and the marbled newt T. marmoratus. The map is based on [6] and updated following recent findings.

and T. pygmaeus, which make up the crested newts' sister group, have the heaviest body build in the genus and possess a typical NRBV count of 12 . Over ninety per cent of these newts can be correctly identified based on NRBV counts alone; interspecific hybridization along parapatric contact zones (Figure 1) is suggested to account for most of the remaining intraspecific variation [2-4]. Crested newt morphology has been interpreted as reflecting phylogeny [2]. A maximally parsimonious interpretation of NRBV (interpreting the ancestral crested newt body shape as relatively robust and the more slender body shapes as derived) suggests a branching order as shown in Figure 2a.

With the advent of molecular techniques, independent data became available and multiple molecular markers have now been employed to attempt to resolve the crested newt phylogeny. Based on restriction fragment length polymorphism of the mitochondrial genome (employing eleven restriction enzymes), a polytomy was found (Figure 2b) [5]. Similarly, analysis of a suite of forty enzyme loci and $642 \mathrm{bp}$ of mitochondrial sequence data resulted in a polytomous relationship for the four morphotypes (Figure 2b) [6]. A polytomy could simply reflect a lack of phylogenetic resolution in the data. However, as the different datasets both pointed towards a polytomy, it was suggested that the four crested newt morphotypes truly split practically simultaneously [6].

A later effort using sequence data of five nuclear DNA (2589 bp) and two mitochondrial DNA markers (1747 bp) revealed a more varied picture [7]. An analysis using the sequence data concatenated found a fully bifurcating and significantly supported phylogeny (Figure 2c). There are, however, theoretical objections to data concatenation, as this method does not consider the unique topological history that each individual gene possesses [8]. Therefore, phylogenetic inference was also carried out using a hierarchical Bayesian analysis, which does explicitly take the effects of gene tree heterogeneity into account. This analysis indeed produced different results. Based on the five nuclear DNA markers, a fourfold polytomy was found again (Figure 2b). This fourfold polytomy could be expanded to a trichotomy by incorporating the mitochondrial DNA data, but the single sister relationship found was incongruent with the data concatenation approach (Figure 2d).

Previous molecular studies have firmly established that the crested newt radiation occurred in a brief time 

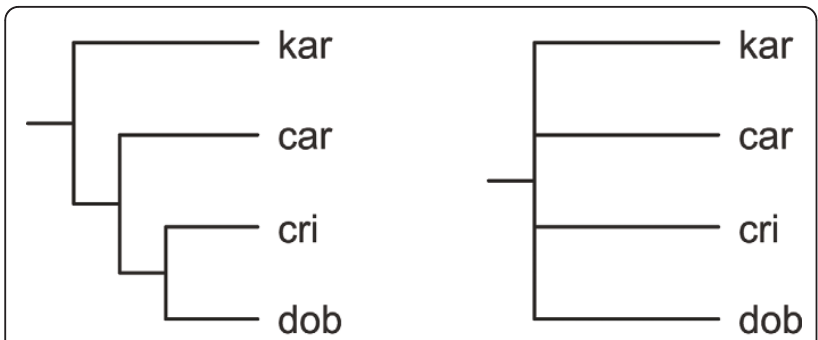

a Morphology DFLP mtDNA
Allozymes mtDNA fragments nucDNA hierarchical Bayesian
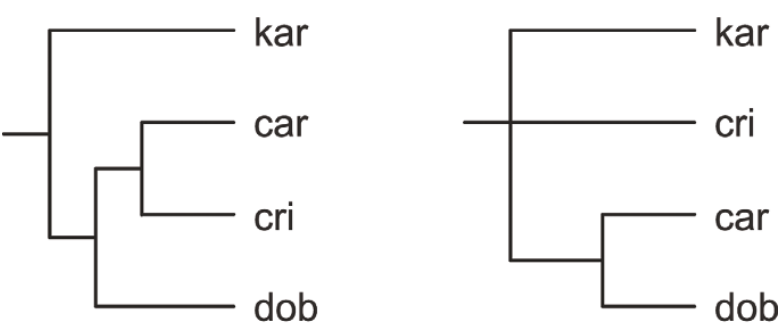

C nucDNA concatenated nucDNA + mtDNA fragments concatenated d nucDNA + mtDNA fragments
hierarchical Bayesian

Figure 2 A summary of the different phylogenetic hypotheses for the four crested newt morphotypes as suggested by previous studies. The datasets supporting each tree are noted below it (see the main text for details and references). Abbreviations used for the four morphotypes are: kar =T. karelinii group; car =

$T$. carnifex - T. macedonicus; $c r i=T$. cristatus; dob $=T$. dobrogicus.

interval. However, they yielded conflicting phylogenetic hypotheses and have been unable to settle the relationship among the morphotypes. Furthermore, all phylogenetic hypotheses found so far are in conflict with the tree suggested by morphology. In this study we further explore the crested newt phylogeny, this time employing complete mitogenomic sequences. As the mitochondrial genome contains tenfold the bp studied up to now ( $\sim 17 \mathrm{Kbp}$ vs. $\sim 1.7 \mathrm{Kbp})$, it is a promising source of phylogenetic resolution (cf. [9]). We here analyse nine newly-sequenced mitogenomes, representing all Triturus species, and manage to obtain a fully-resolved crested newt phylogeny. We discuss this new phylogeny with respect to previous attempts to obtain the Triturus tree and speculate on causes for cladogenesis.

\section{Results}

We present a mitogenomic Triturus phylogeny (Figure 3) based on a division of the mitogenomic sequence data into 42 data partitions, as this partitioning strategy is preferred over the simpler ones tested (Table 1). Bayesian inference identifies a basal dichotomy in the crested newt superspecies between the T. karelinii group and the other morphotypes (node I in Figure 3). The next bifurcation divides the latter assortment in
T. carnifex - T. macedonicus (node II) versus T. cristatus and T. dobrogicus (node III). Posterior probabilities are $\geq 0.95$ (Table 2). Although Maximum Likelihood inference yields the same branching order and similar branch lengths (tree not shown), the bootstrap support values for two of the three nodes associated with radiation of crested newt morphotypes (nodes II and III) are low (Table 2). The mitogenomic phylogeny is characterized by long terminal branches, which are connected by short internal branches. The three nodes connecting the crested newt morphotypes represent a narrow time window (approximately 10.4-8.8 Ma) and have small confidence intervals, independent of dating method used (Table 2). Three character state transitions are required to explain the NRBV differentiation across the four crested newt morphotypes, two of which are situated on short internal branches (Figure 3).

\section{Discussion}

\section{The mitogenomic Triturus tree}

The full mitogenomic dataset has provided the phylogenetic resolution that the crested newt case required. Previous approaches using only part of the mitochondrial genome found a polytomy for the four morphotypes, but based on the full mitochondrial DNA sequences we managed to resolve this polytomy. Under Bayesian inference, we find a fully bifurcating phylogeny, with significant support for the three nodes connecting the four crested newt morphotypes (i.e. nodes I-III in Figure 3 and Table 2). In comparison, Maximum Likelihood bootstrapping finds equivocal results for two of the three nodes (nodes II and III). Disparity in support values between both methods is known to occur at short internodes, where Bayesian inference appears to better exploit the relatively small number of informative characters [10]. Our confidence in the Bayesian phylogeny is increased by its correspondence to the branching order suggested by a maximally parsimonious interpretation of NRBV evolution (Figure 2a and 3).

The mitochondrial genome, given its non-recombining nature, behaves as a single gene and, due to stochasticity in the coalescent process, the branching order it suggests is not necessarily congruent with the true species tree [11]. The motivation behind studying independent gene trees (i.e. multiple nuclear genetic markers) is that these should ultimately converge upon the overarching species tree [8]. However, a recent hierarchical Bayesian analysis based on five nuclear markers did not yield a resolved crested newt phylogeny [7]. This lack of resolution could be explained by the rapidness of the radiation of the crested newt morphotypes, as repeated cladogenesis within the temporal domain of the lineage sorting process increases the chance of a mismatch between gene trees and species tree $[1,8,11-13]$. Such a risk of gene tree - species tree discordance is smaller for the mitochondrial genome, because 


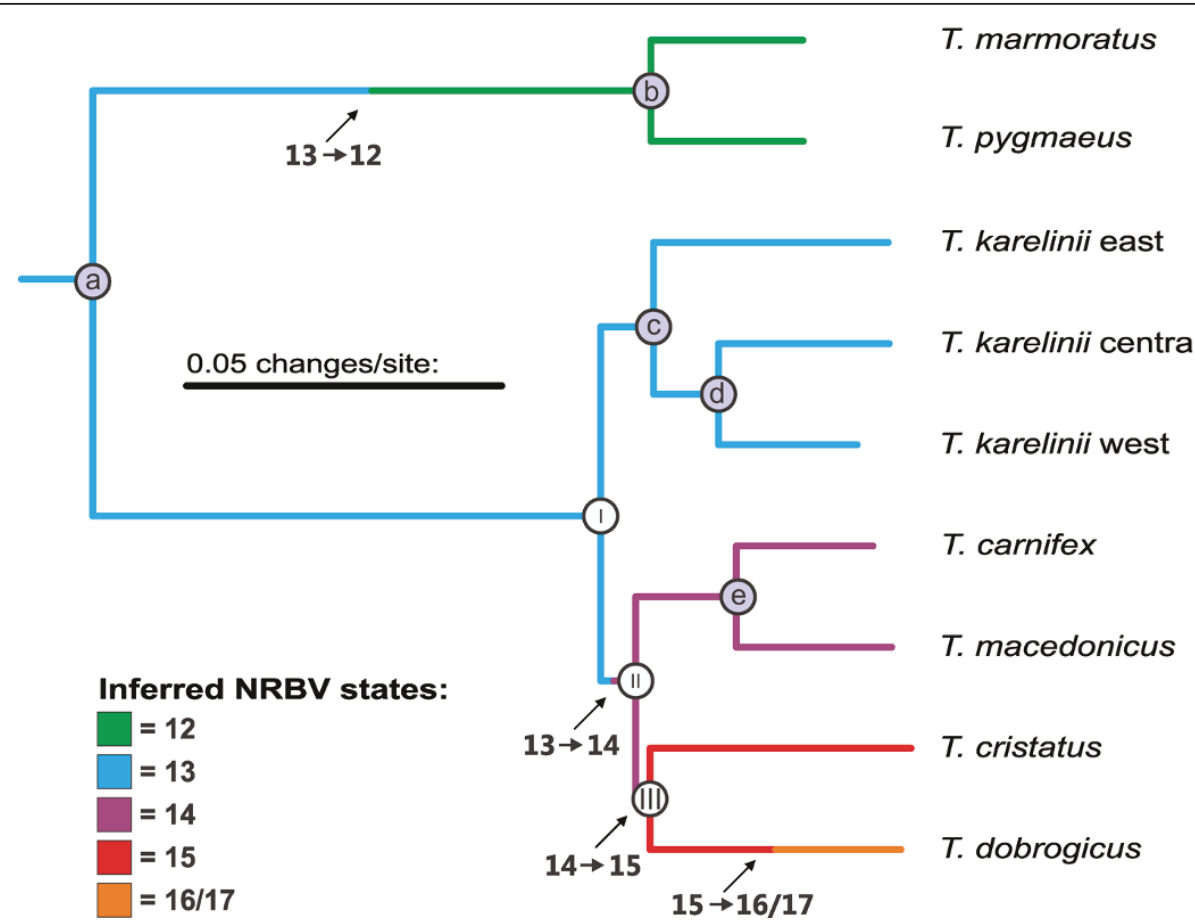

Figure 3 The mitogenomic Triturus tree resulting from the Bayesian inference. The Calotriton asper outgroup is not shown. Nodes are coded and correspond to table 2; the nodes of interest, separating the crested newt morphotypes, are coded I-III (the remaining nodes are coded a-e). The NRBV additions required to explain the NRBV variation observed in Triturus today are noted along the phylogeny (interpreting NRBV $=13$ as the ancestral character state, see Additional File 1). The exact timing of inferred NRBV shifts is not known, only that they are positioned on a particular branch.

lineage sorting is realized faster compared to the nuclear genome (given the fourfold smaller effective population size of the mitochondrial genome due to haploid and uniparental inheritance $[14,15])$.

We do not claim that our current attempt resolves the crested newt species tree once and for all; studying a much larger battery of nuclear DNA markers than previously used is required to further home in on the crested newt species tree [12]. However, considering the data currently available, we suggest that the Bayesian mitogenomic phylogeny as yet provides the most reliable estimation of the crested newt species tree. We here

Table 1 Evaluation of the optimal partitioning scheme for the mitogenomic sequence data based on Bayes factor analysis

\begin{tabular}{rrrrrr}
\hline & $\mathbf{4 2}$ & $\mathbf{6}$ & $\mathbf{2 9}$ & $\mathbf{1 6}$ \\
\hline 16 & $5,300.00$ & $4,554.12$ & $4,553.18$ & - \\
29 & 746.82 & 0.94 & - & - \\
6 & 745.89 & - & - & - \\
42 & - & - & - & -
\end{tabular}

A pairwise comparison of the four tested partitioning strategies $(6,16,29$ or 42 data partitions), which are ordered from highest to lowest marginal likelihood from left to right and vice versa from top to bottom. The marginal likelihoods, in order from highest to lowest, are: $42=-48316.02,6=$ $-48688.96,29=-48689.43$ and $16=-50966.02$. employ the new phylogeny to explore the potential causes underlying the splits between the crested newt morphotypes.

The potential of paleogeography to explain crested newt speciation

Based on temporal estimates associated with the crested newt splits (Table 2), potential vicariant events can be identified by consulting paleogeographic reconstructions.

Table 2 Support values and temporal estimates for Triturus nodes

\begin{tabular}{lcccccc}
\hline & \multicolumn{2}{c}{ Support values } & \multicolumn{2}{c}{ Dating r8s } & \multicolumn{2}{c}{ Dating BEAST } \\
Node & MrBayes & RAxML & Mean & $\mathbf{9 5 \%} \mathbf{C l}$ & Mean & $\mathbf{9 5 \%} \mathbf{~ C l}$ \\
\hline I & 1.0 & 100 & 10.3 & $9.4-11.2$ & 10.4 & $9.4-11.5$ \\
II & 1.0 & 62 & 9.2 & $8.5-9.4$ & 9.3 & $8.3-10.2$ \\
III & 0.95 & 46 & 8.7 & $8.0-9.4$ & 8.8 & $7.8-9.7$ \\
a \# & 1.0 & 100 & 28.0 & $24.9-31.1$ & 27.6 & $24.8-30.8$ \\
b & 1.0 & 100 & 5.9 & $5.0-6.8$ & 5.6 & $4.7-6.6$ \\
C & 1.0 & 100 & 8.3 & $7.3-9.3$ & 8.3 & $7.3-9.4$ \\
d & 1.0 & 100 & 5.8 & $5.1-6.6$ & 5.8 & $5.0-6.7$ \\
e \# & 1.0 & 100 & 5.33 & $n / a$ & 5.33 & $n / a$ \\
\hline The coded nodes correspond to Figure 2. The nodes of interest, separating \\
the crested newt morphotypes, are coded I-III (the remaining nodes are coded \\
a-e). Nodes marked with a \# are used for temporal calibration.
\end{tabular}


We here concentrate on the three splits which gave rise to the four crested newt morphotypes (i.e. nodes I-III in Figure 3); for vicariant events underlying the three splits within morphotypes (i.e. nodes c-e in Figure 3), see $[6,16]$. An earlier attempt to reconstruct the historical biogeography of crested newts assumed a 'hard' polytomous relationship for the four morphotypes [6]. In effect, a temporal estimate was only provided for the crown of the crested newts and proposed vicariant events were derived from this date.

The present study has resolved the relationships among the morphotypes (i.e. the polytomy in [6] turned out to be 'soft' after all). In line with the increased phylogenetic resolution, more recent dates are appointed to the newly resolved nodes and their morphotype lineages. The crested newt crown, i.e. the split between the T. karelinii group and the remaining crested newts (node I in Figure 3), is dated at ca. 10.4 Ma. The origin of the Aegean Sea at 12-9 Ma [17], which separated the Balkan Peninsula from Anatolia, is a likely underlying vicariant event (sensu [6]). No obvious vicariant events can be associated with the two splits that gave rise to the three remaining morphotypes [18]: the separation of T. carnifex - T. macedonicus versus T. cristatus and T. dobrogicus (node II) at approximately 9.3 Ma and the split between $T$. cristatus and $T$. dobrogicus (node III) around 8.8 Ma (contra [6]).

No comprehensive paleogeographical reconstructions are as yet available for the Balkan Peninsula in the period between 11 and $8.5 \mathrm{Ma}$ [18]. It is feasible that vicariant events relevant to the crested newt case have yet to be discovered. As a way forward, we suggest that more taxa with Balkan distributions should be surveyed in a historical biogeographical context $[6,19]$. By uncovering congruent spatio-temporal signatures, such studies should assist in paleogeographical reconstruction of the Balkan Peninsula. This strategy might not be so straightforward; genetic structuring in the two other groups of newts that occur on the Balkan Peninsula (Ichthyosaura and Lissotriton), though relatively old, originated considerably more recent than that in the crested newts [20,21].

\section{Could ecological divergence have played a role in crested newt speciation?}

The role of ecological divergence in historical biogeography is often regarded as passive; external factors such as geology and climate are considered to be responsible for the actual dividing of ancestral stocks and potential ecological divergence occurs at a later point [22]. However, ecology can play an active role in the speciation process: disruptive selection along an ecological gradient can result in restrictions to gene flow, in the absence of geographical isolation [22-25]. Could such a parapatric mode of speciation apply to the crested newt case?
The crested newt morphotypes do show ecological differentiation in the time they annually spend in the water. The duration of the annual aquatic period is three months in the T. karelinii group, four in T. carnifex, five in T. cristatus and six in T. dobrogicus [4]. Crested newts thus show a correlation between phenotype and phenology: sturdy bodies and a low NRBV count are associated with a more terrestrial way of life and slender bodies and a high NRBV count with a more aquatic life style [4]. This notion of a terrestrial versus aquatic trade-off associated with body shape is further supported by the even more robust marbled newt $T$. marmoratus, whose two-month aquatic phase is the shortest of the Triturus newts [6].

The body shape differentiation in crested newts occurred over a brief timespan (Figure 3 and Table 2): at least two of the three NRBV additions during crested newt evolution are associated with short internal branches. Under the assumption that today's phenology-phenotype correlation has been valid through time, the ecological divergence of the crested newt morphotypes must have similarly taken place over a short period. Considering the fundamental role water bodies play in amphibian reproduction [26], it is reasonable to suggest that differences in the availability of standing water would present different adaptive peaks. The time frame of the crested newt radiation corresponds to a period of increased seasonality in Eurasia during the Late Miocene (11.6-5.3 Ma), associated with the uplift of the Tibetan Plateau, which led to a more heterogeneous landscape in terms of humidity [27].

With a clear speciation scenario involving vicariance lacking, we, as an alternative hypothesis, suggest that the crested newts' body shape differentiation reflects a rapid adaptive radiation to different water regimes. It should be noted that these two hypotheses are not necessarily mutually exclusive.

\section{Reflections on NRBV and its adaptive value in Triturus}

Salamanders have become a model group to understand patterns of morphological evolution [28]. Variation of salamander body shape has been accomplished by modifying the vertebral column, by either altering the length of the individual vertebrae or by changing the total number of vertebrae [29]. Interestingly, the intrageneric range in NRBV count shown by Triturus is unprecedented in Salamandridae (see Additional file 1), suggesting body shape plasticity played a prominent role during crested newt evolution. Work on the genetic pathways underlying the evolutionary development of the different Triturus morphotypes will provide more insight into this phenomenon (J.M. Ziermann et al., in prep.).

What could be the adaptive value of the crested newt NRBV radiation? The dualism of an amphibian lifestyle poses conflicting demands on body shape. The rapid adaptive radiation scenario we propose for the crested 
newts, suggests that the balance struck for this trade-off differs among the morphotypes, due to the different ecological background each of them experiences. What do we currently understand about the differential performance of the morphotypes in the aquatic and the terrestrial environment? Although [30] found that Triturus stockiness is positively correlated with running speed, this is not so for slenderness and swimming speed. What could be the reason for this partially unexpected result? Maybe 'speed' is not the most suitable way to characterize terrestrial or aquatic specialization? Perhaps body elongation benefits more aquatic Triturus newts some other way, e.g. by increasing maneuverability or by providing more space for egg production [30]? More research is required to elucidate the adaptive value of the different Triturus body shapes against distinct ecological backgrounds.

\section{Conclusions}

Although it has proven difficult to resolve the rapid radiation of crested newts, by employing full mitochondrial DNA sequence data we now have a precise estimate of the chronology of branching events. The relationship among the four crested newt morphotypes found agrees with a maximally parsimonious interpretation of NRBV evolution, increasing our confidence in the accuracy of the branching order. The basal dichotomy sorting out the crested newt morphotypes can be associated with a major vicariant event, but we cannot pinpoint drivers for the other two splits sorting out the morphotypes. We propose that (as yet) undiscovered vicariant events and/or ecological divergence (reflected by body shape differentiation) resulted in a disruption of gene flow. Crested newts are a suitable model to study eco-evo-devo in a rapid radiation and the new phylogenetic framework presented here serves as a baseline for future research.

\section{Methods}

\section{Samples}

We included seven crested newts (see Additional file 2), representing all recognized species, as well as three distinct mitochondrial DNA clades that constitute the $T$. karelinii group (cf. [16]). We follow [16] in awaiting a taxonomic revision of the $T$. karelinii group before applying specific names to the three constituent mitochondrial DNA clades (the name T. karelinii sensu stricto would apply to the 'eastern clade' and T. arntzeni has been applied to the 'western clade'; no name has as yet been proposed for the 'central clade'). We also sequenced the two marbled newts (T. marmoratus and T. pygmaeus), the remaining members of the genus Triturus, to function as outgroup taxa. Additionally, we added a sequence of Calotriton asper, sister to the genus Triturus, available from [31] (GenBank accession number EU880307).

\section{Sequences}

The complete mitogenomes of the nine Triturus newts were sequenced in fifteen overlapping parts. We followed the laboratory protocol of [31] and designed more specific and/or internal primers where required (detailed in Additional file 3). Cycle sequencing was done commercially through Macrogen Inc. Forward and reverse sequences were checked by eye and consensus sequences were compiled with Sequencher 4.5 (Gene Codes Corporation). The fifteen fragments per individual were manually aligned and merged in MacClade 4.08 [32]. The length of the resulting sequences ranged from 16,424 to $16,649 \mathrm{bp}$. The Triturus mitogenomes are composed of thirteen protein-coding genes, twenty-two transfer RNA genes, two ribosomal RNA genes, the D-loop, and a non-coding region (which is highly variable in length) and gene order is identical to that found in the rest of the family Salamandridae (cf. [31]). The newly produced mitogenomic sequences have been submitted to GenBank (accession numbers are noted in Additional file 2). The $15420 \mathrm{bp}$ data matrix used for phylogenetic analyses, comprising the ribosomal RNA, transfer RNA and protein-coding genes, is available from TreeBASE (study ID S11081).

\section{Data partitioning}

We compared four different partitioning strategies for organizing the mitochondrial sequence data (cf. [33]). Each partitioning strategy treated the two ribosomal RNA genes and the concatenated transfer RNA genes as separate partitions. Differences among the partitioning strategies are based on the treatment of the protein-coding genes, dividing the protein-coding data according to: first, second and third codon position (6 partitions in total), each gene (16 partitions); first plus second and third codon position for each gene (29 partitions); and first, second and third codon position for each gene (42 partitions). The most appropriate model of sequence evolution for each data partition was determined with MrModeltest 2.2 [34], based on the Akaike Information Criterion (see Additional file 4). The optimal partitioning scheme was selected based on the differences in the harmonic mean of the -ln likelihood scores resulting from the Bayesian phylogenetic inference. The 2 ln Bayes factors were calculated for each partitioning strategy by subtracting the score resulting from simpler partitioning strategies and multiplying the outcome by -2 [35,36]. A value for 2 ln Bayes factor exceeding 10 was used as a threshold for preferring the more complex model [37].

\section{Phylogenetic analyses}

Mixed-model Bayesian phylogenetic inference was carried out with MrBayes 3.1.2 [38]. For each data partition the rate of sequence evolution and parameters were unlinked. Two simultaneous four chain runs proceeded 
for one hundred million generations, with a sampling frequency of 0.001 and a heating parameter of 0.05 . The first half of the sampled trees was discarded as burn-in and the phylogenetic inference was drawn from the remaining 'forest'. Tracer [39] was used to check for stabilization of overall likelihood within and convergence between runs. Partitioned Maximum Likelihood phylogenetic inference was carried out with RAxML 7.2.7 [40]. Robustness of the tree was tested via 100 bootstrap replicates. All phylogenetic analyses were carried out via the CIPRES Science Gateway [41].

\section{Temporal calibration}

We used two independent calibration points for molecular dating, one fossil-based and one geology-based (cf. [16]). A fossil dated at $24 \mathrm{Ma}$ was interpreted as a minimum estimate for the most recent common ancestor of the genus Triturus (cf. [42]) and the origin of the Adriatic Sea at $5.33 \mathrm{Ma}$, at the end of the Messinian Salinity Crisis, was interpreted as the vicariant event causing the T. carnifex - T. macedonicus split (cf. [6]). Divergence times were estimated with r8s 1.71 [43] and BEAST 1.5.3 [44]. In r8s we used the penalized-likelihood approach in combination with the truncated-Newton algorithm. Calotriton asper was pruned from the dataset, while keeping the root position, to avoid performing the time estimation on a basal trichotomy. The optimal smoothing parameter $(S=1)$ was determined by a cross-validation procedure, using the Bayesian consensus tree as input. Mean temporal estimates and 95\% confidence intervals were determined by profiling the last thousand sampled Bayesian topologies. In BEAST, we applied the uncorrelated lognormal relaxed clock model and a Yule speciation model. The fixed calibration point was appointed a normally distributed prior with a small standard deviation (0.001) and the minimum estimate a lognormally distributed prior with the default standard deviation (1.0). The tree resulting from the Bayesian analysis was used as starting topology. Each data partition was allowed its own model of sequence evolution, as previously determined with MrModeltest. Divergence times were estimated based on two independent 100 million generation runs, sampled every 1000 generations, after discarding the first half of generations as burn-in. Tracer [39] was used to check whether effective sample sizes were at least 200.

\section{Additional material}

Additional file 1: NRBV tracing for the Salamandridae. By tracing NRBV evolution over a phylogeny of the Salamandridae, the ancestral NRBV state for Triturus is determined.

Additional file 2: Sampling details. The Triturus material included in this study.
Additional file 3: Primer information. The primers used to sequence the Triturus mitochondrial genomes.

Additional file 4: Models of sequence evolution. Models of sequence evolution for different data partitions as suggested by MrModeltest.

\section{Acknowledgements}

G. Wallis and two reviewers provided valuable comments on an earlier version of this manuscript.

\section{Author details}

${ }^{1}$ Netherlands Centre for Biodiversity - NCB Naturalis, P. O. Box 9517, 2300 RA Leiden, The Netherlands. ${ }^{2}$ International Institute for Geo-Information Science and Earth Observation - ITC, P.O. Box 6, 7500 AA, Enschede, The Netherlands.

\section{Authors' contributions}

BW conducted laboratory work. BW and JWA analyzed the data and wrote the manuscript. All authors read and approved the final manuscript.

Received: 20 March 2011 Accepted: 14 June 2011

Published: 14 June 2011

\section{References}

1. Whitfield JB, Lockhart PJ: Deciphering ancient rapid radiations. Trends Ecol Evol 2007, 22(5):258-265.

2. Arntzen JW, Wallis GP: Geographic variation and taxonomy of crested newts (Triturus cristatus superspecies): morphological and mitochondrial data. Contrib Zool 1999, 68(3):181-203.

3. Arntzen JW, Wallis GP: The 'Wolterstorff Index' and its value to the taxonomy of the crested newt superspecies. Abhandlungen und Berichte für Naturkunde 1994, 17:57-66.

4. Arntzen JW: Triturus cristatus Superspecies-Kammolch-Artenkreis. In Handbuch der Reptilien und Amphibien Europas Schwanzlurche IIA. Edited by: Grossenbacher K, Thiesmeier B. Wiebelsheim: Aula-Verlag; 2003:421-514.

5. Wallis GP, Arntzen JW: Mitochondrial-DNA variation in the crested newt superspecies: limited cytoplasmic gene flow among species. Evolution 1989, 43(1):88-104.

6. Arntzen JW, Espregueira Themudo G, Wielstra B: The phylogeny of crested newts (Triturus cristatus superspecies): nuclear and mitochondrial genetic characters suggest a hard polytomy, in line with the paleogeography of the centre of origin. Contrib Zool 2007, 76(4):261-278.

7. Espregueira Themudo G, Wielstra B, Arntzen JW: Multiple nuclear and mitochondrial genes resolve the branching order of a rapid radiation of crested newts (Triturus, Salamandridae). Mol Phylogenet Evol 2009, 52(2):321-328.

8. Edwards SV: Is a new and general theory of molecular systematics emerging? Evolution 2009, 63(1):1-19.

9. Cummings MP, Otto SP, Wakeley J: Sampling properties of DNA-sequence data in phylogenetic analysis. Mol Biol Evol 1995, 12(5):814-822.

10. Alfaro ME, Zoller S, Lutzoni F: Bayes or bootstrap? A simulation study comparing the performance of Bayesian Markov chain Monte Carlo sampling and bootstrapping in assessing phylogenetic confidence. $\mathrm{Mol}$ Biol Evol 2003, 20(2):255-266.

11. Degnan JH, Rosenberg NA: Gene tree discordance, phylogenetic inference and the multispecies coalescent. Trends Ecol Evol 2009, 24(6):332-340.

12. Maddison WP, Knowles LL: Inferring phylogeny despite incomplete lineage sorting. Syst Biol 2006, 55(1):21-30.

13. Moore WS: Inferring phylogenies from mtDNA variation - mitochondrialgene trees versus nuclear-gene trees. Evolution 1995, 49(4):718-726.

14. Ballard JWO, Whitlock MC: The incomplete natural history of mitochondria. Mol Ecol 2004, 13(4):729-744.

15. Funk DJ, Omland KE: Species-level paraphyly and polyphyly: frequency, causes, and consequences, with insights from animal mitochondrial DNA. Annu Rev Ecol Evol Syst 2003, 34:397-423.

16. Wielstra B, Espregueira Themudo G, Güclü Ö, Olgun K, Poyarkov NA, Arntzen JW: Cryptic crested newt diversity at the Eurasian transition: the mitochondrial DNA phylogeography of Near Eastern Triturus newts. Mol Phylogenet Evol 2010, 56(3):888-896. 
17. Dermitzakis DM, Papanikolaou DJ: Paleogeography and geodynamics of the Aegean region during the Neogene. Annales Géologiques des Pays Helléniques 1981, 30:245-289.

18. Popov SV, Rögl F, Rozanov AY, Steiniger FF, Shcherba IG, Kovac M: Lithological-paleogeographic maps of Paratethys: 10 maps Late Eocene to Pliocene. Courier Forschunginstitut Senckenberg 2004, 250:1-46.

19. Arbogast BS, Kenagy GJ: Comparative phylogeography as an integrative approach to historical biogeography. J Biogeogr 2001, 28(7):819-825.

20. Babik W, Branicki W, Crnobrnja-Isailovic J, Cogalniceanu D, Sas I, Olgun K, Poyarkov NA, Garcia-Paris M, Arntzen JW: Phylogeography of two European newt species- discordance between mtDNA and morphology. Mol Ecol 2005, 14(8):2475-2491.

21. Sotiropoulos K, Eleftherakos K, Dzukic G, Kaiezic ML, Legakis A, Polymeni RM: Phylogeny and biogeography of the alpine newt Mesotriton alpestris (Salamandridae, Caudata), inferred from mtDNA sequences. Mol Phylogenet Evol 2007, 45(1):211-226.

22. Coyne JA, Orr HA: Speciation. Sunderland: Sinauer Associates; 2004.

23. Schluter D: Ecology and the origin of species. Trends Ecol Evol 2001, 16(7):372-380.

24. Rundle HD, Nosil P: Ecological speciation. Ecol Lett 2005, 8(3):336-352.

25. Sobel JM, Chen GF, Watt LR, Schemske DW: The biology of speciation. Evolution 2010, 64(2):295-315.

26. Carey C, Alexander MA: Climate change and amphibian declines: is there a link? Diversity Distrib 2003, 9(2):111-121.

27. Eronen JT, Ataabadia MM, Micheelsb A, Karme A, Bernor RL, Fortelius M: Distribution history and climatic controls of the Late Miocene Pikermian chronofauna. Proc Natl Acad Sci USA 2009, 106(29):11867-11871.

28. Wake DB: What salamanders have taught us about evolution. Annu Rev Ecol Evol Syst 2009, 40:333-352.

29. Parra-Olea G, Wake DB: Extreme morphological and ecological homoplasy in tropical salamanders. Proc Natl Acad Sci USA 2001, 98(14):7888-7891.

30. Gvozdík L, van Damme R: Triturus newts defy the running-swimming dilemma. Evolution 2006, 60(10):2110-2121.

31. Zhang P, Papenfuss TJ, Wake MH, Qu LH, Wake DB: Phylogeny and biogeography of the family Salamandridae (Amphibia: Caudata) inferred from complete mitochondrial genomes. Mol Phylogenet Evol 2008, 49(2):586-597.

32. Maddison DR, Maddison WP: MacClade 4: analysis of phylogeny and character evolution, version 4.08. Sunderland (Massachusetts): Sinauer Associates 2005.

33. Mueller RL, Macey JR, Jaekel M, Wake DB, Boore JL: Morphological homoplasy, life history evolution, and historical biogeography of plethodontid salamanders inferred from complete mitochondrial genomes. Proc Natl Acad Sci USA 2004, 101(38):13820-13825.

34. Nylander JAA: MrModelTest2.[http://www.abc.se/ nylander].

35. Brandley MC, Schmitz A, Reeder TW: Partitioned Bayesian analyses, partition choice, and the phylogenetic relationships of scincid lizards. Syst Biol 2005, 54(3):373-390.

36. Nylander JAA, Ronquist F, Huelsenbeck JP, Nieves-Aldrey JL: Bayesian phylogenetic analysis of combined data. Syst Biol 2004, 53(1):47-67.

37. Kass RE, Raftery AE: Bayes factors. J Amer Statistical Assoc 1995, 90(430):773-795

38. Ronquist F, Huelsenbeck JP: MrBayes 3: Bayesian phylogenetic inference under mixed models. Bioinformatics 2003, 19(12):1572-1574.

39. Rambaut A, Drummond AJ: Tracer v1.4.[http://beast.bio.ed.ac.uk/tracer].

40. Stamatakis A: RAxML-VI-HPC: Maximum likelihood-based phylogenetic analyses with thousands of taxa and mixed models. Bioinformatics 2006, 22(21):2688-2690.

41. Miller MA, Holder MT, Vos R, Midford PE, Liebowitz T, Chan L, Hoover P, Warnow T: The CIPRES Portals.[http://www.phylo.org/sub_sections/portal].

42. Steinfartz S, Vicario S, Arntzen JW, Caccone A: A Bayesian approach on molecules and behavior: reconsidering phylogenetic and evolutionary patterns of the Salamandridae with emphasis on Triturus newts. J Exp Zool B: Mol Dev Evol 2007, 308B(2):139-162.

43. Sanderson MJ: r8s: inferring absolute rates of molecular evolution and divergence times in the absence of a molecular clock. Bioinformatics 2003, 19(2):301-302.

44. Drummond AJ, Rambaut A: BEAST: Bayesian evolutionary analysis by sampling trees. BMC Evol Biol 2007, 7:214 doi:10.1186/1471-2148-11-162

Cite this article as: Wielstra and Arntzen: Unraveling the rapid radiation of crested newts (Triturus cristatus superspecies) using complete mitogenomic sequences. BMC Evolutionary Biology 2011 11:162.

\section{Submit your next manuscript to BioMed Central and take full advantage of:}

- Convenient online submission

- Thorough peer review

- No space constraints or color figure charges

- Immediate publication on acceptance

- Inclusion in PubMed, CAS, Scopus and Google Scholar

- Research which is freely available for redistribution

Submit your manuscript at www.biomedcentral.com/submit
Ciomed Central 